\title{
A Unified Approach for the Identification of SISO/MIMO Wiener and Hammerstein Systems *
}

\author{
Fayçal Ikhouane* F. Giri ${ }^{* *}$ \\ * Universitat Politècnica de Catalunya, Escola Universitària \\ d'Enginyeria Tècnica Industrial de Barcelona, Departament de \\ Matemàtica Aplicada III, Comte d'Urgell, 187, 08036, Barcelona, \\ Spain.(faycal.ikhouane@upc.edu) \\ ** GREYC laboratory, University of Caen, France. \\ (fouad.giri@unicaen.fr)
}

\begin{abstract}
Hammerstein and Wiener models are nonlinear representations of systems composed by the coupling of a static nonlinearity $\mathrm{N}$ and a linear system $\mathrm{L}$ in the form $\mathrm{N}-\mathrm{L}$ and L-N respectively. These models can represent real processes which made them popular in the last decades. The problem of identifying the static nonlinearity and linear system is not a trivial task, and has attracted a lot of research interest. It has been studied in the available literature either for Hammerstein or Wiener systems, and either in a discrete-time or continuous-time setting. The objective of this paper is to present a unified framework for the identification of these systems that is valid for SISO and MIMO systems, discrete and continuous-time setting, and with the only a priori knowledge that the system is either Wiener or Hammerstein.
\end{abstract}

Keywords: Nonlinear system identification, Wiener systems, Hammerstein systems.

\section{INTRODUCTION}

Hammerstein and Wiener models are block-oriented models composed by the coupling of a static nonlinearity $\mathrm{N}$ and a linear system $\mathrm{L}$ in the form N-L and $\mathrm{L}-\mathrm{N}$ respectively. Although simple, these structures can capture the behavior of many real-life processes $[4,15,8]$. The problem of identifying the static nonlinearity $\mathrm{N}$ and linear system $\mathrm{L}$ from input-output data has attracted a lot of research interest, and many methods are available for this problem as early as [16]. In [13], sine waves are used to excite a SISO $^{1}$ Wiener continuous-time system, and frequency techniques are used to determine the unknowns. Frequency techniques for SISO Wiener systems is also used in [3]. In [5] a MIMO $^{2}$ Hammerstein discrete-time system is considered with a nonlinearity $\mathrm{N}$ that has a special structure in terms of cardinal spline functions. In [6] a discretetime Wiener system with a special class of nonlinearities is considered. In [9] a discrete-time Hammerstein system with a special class of nonlinearities is considered. Other references include $[10,17,19,22,7]$. A recent overview of previous works may be found in [11].

The objective of this paper is to present a unified framework for the identification of Wiener and Hammerstein systems. This framework is valid for SISO and MIMO systems, continuous and discrete-time systems. The nonlinear static function is a linear combination of known functions

\footnotetext{
* Supported by grant DPI2011-25822 of the Spanish Ministry of Science and Innovation.

1 Single input single output

2 Multi-input multi-output
}

with unknown multiplying parameters. It is assumed that the linear system has an invertible static gain matrix. The identification methodology consists in three stages:

(1) The first stage consists in determining the nonlinear static function using constant input signals and using the fact that the linear system is asymptotically stable.

(2) The second stage consists in transforming the nonlinear Hammerstein or Wiener identification problem into a linear identification problem that can be solved using standard techniques. This is done by assuming that the nonlinearity is a local homeomorphism, so that it can be locally inverted.

(3) The third stage consists in performing a validation test on the previously obtained (Wiener and Hammerstein) models, and retaining the one that passes better this test.

The main features of the proposed identification approach are:

(1) It is not needed to know a priori whether the system is Wiener or Hammerstein. It is only required that the system belongs to the set $\{$ Wiener, Hammerstein $\}$.

(2) Both SISO and MIMO, continuous- and discrete-time systems are simultaneously handeled within the same framework.

This paper is organized as follows. Section 2 presents the identification problem under study and introduces the assumptions needed to solve this problem. Section 3 presents 
and analyzes the identification algorithm in the absence of noise and Section 4 analyzes the identification algorithm in the presence of noise. Conclusions are presented in Section 5 .

\section{PROBLEM FORMULATION}

Consider the linear system:

$$
\begin{aligned}
\bar{x}(t) & =A x(t)+B \vartheta(t) \\
\theta(t) & =C x(t)+D \vartheta(t) \\
x(0) & =x_{0} \in \mathbb{R}^{p}
\end{aligned}
$$

where $\bar{x}=\frac{\mathrm{d} x}{\mathrm{~d} t}$ for continuous-time systems and $\bar{x}(t)=$ $x(t+1)$ for discrete-time systems. We consider that $A \in$ $\mathbb{R}^{p \times p}$ is Hurwitz, ${ }^{3} B \in \mathbb{R}^{p \times n}, C \in \mathbb{R}^{n \times p}, D \in \mathbb{R}^{n \times n}$. For continuous-time systems we have $t \in \mathbb{R}_{+}$and $\vartheta \in$ $L^{\infty}\left(\mathbb{R}_{+}, \mathbb{R}^{n}\right)^{4}$ so that $x \in W^{1, \infty}\left(\mathbb{R}_{+}, \mathbb{R}^{p}\right)^{5}$ and $\theta \in$ $L^{\infty}\left(\mathbb{R}_{+}, \mathbb{R}^{n}\right)$. For discrete-time systems we have $t \in \mathbb{N}$, and the sets $\{\vartheta(t), t \in \mathbb{N}\},\{\theta(t), t \in \mathbb{N}\},\{x(t), t \in \mathbb{N}\}$ are bounded.

Note that since the matrix $A$ is Hurwitz, it is invertible for continuous-time systems and $\mathcal{I}_{p}-A$ is invertible for discrete-time systems where $\mathcal{I}_{p}$ is the identity matrix of $\mathbb{R}^{p \times p}$. The static gain of the linear system (1)-(2) is given by the matrix $G=-C A^{-1} B+D$ for continuous-time systems and by $G=C\left(\mathcal{I}_{p}-A\right)^{-1} B+D$ for discrete-time systems.

Assumption 1. The matrix $G$ is invertible.

Remark 1. Assumption 1 only excludes pure derivative systems which are rarely met in real-life situations.

We consider a continuous function $f: E \triangleq \prod_{i=1}^{n}\left[-a_{i}, a_{i}\right] \subset$ $\mathbb{R}^{n} \rightarrow \mathbb{R}^{n}$ where $a_{i}>0, i=1, \ldots, n$, that can be written in following way. There exist a known $N \in \mathbb{N},{ }^{6}$ known functions $\Psi_{i}: E \rightarrow \mathbb{R}^{n}, i=1, \ldots, N$ such that

$$
\forall x \in E, f(x)=\sum_{i=1}^{N} \rho_{i} \Psi_{i}(x)
$$

where the $\rho_{i}$ 's are unknown parameters to be determined. These functions $\Psi_{i}, i=1, \ldots, N$ are chosen in such a way that the knowledge of $f(x)$ for some finite number of values $x$ gives the values of the unknown parameters $\rho_{i}, i=1, \ldots, N$. For example when the $\Psi_{i}$ 's are scalar

\footnotetext{
3 That is such that all its eigenvalues have negative real part for continuous-time systems, and with eigenvalues inside the open unit disc for discrete-time systems.

4 This is the Banach space of essentially bounded Lebesgue measurable functions [18].

5 The Sobolev space $W^{1, \infty}\left(\mathbb{R}_{+}, \mathbb{R}^{n}\right)$ is the Banach space of absolutely continuous functions $u: \mathbb{R}_{+} \rightarrow \mathbb{R}^{n}$, where $n$ is a positive integer. For this class of functions, the derivative $\dot{u}$ is Lebesgue measurable and is equal a.e. to the weak derivative of $u$. Moreover, we have $\|u\|_{\infty}<\infty$ and $\|\dot{u}\|_{\infty}<\infty[1]$.

6 In fact, only an upper bound on $N$ is needed.
}

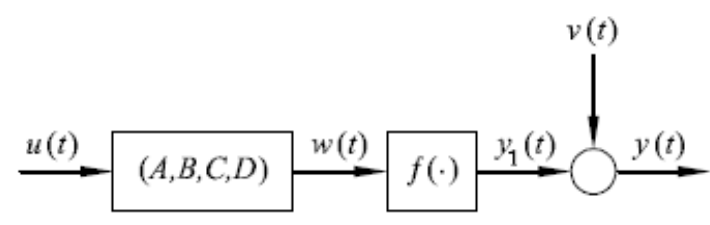

Fig. 1. Wiener system.

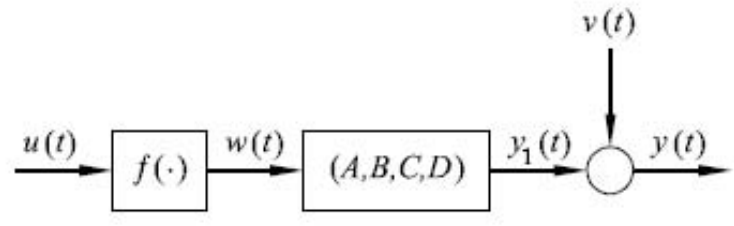

Fig. 2. Hammerstein system.

polynomials, the inversion of the so-called Vandermonde matrix gives the unknown parameters [12]. The $\Psi_{i}$ 's may also constitute a set of orthogonal functions (e.g. Laguerre polynomials). In this case, the unknown parameters may be recovered using least square estimators.

Assumption 2. There exists a (possibly unknown) $\zeta=$ $\left(\zeta_{1}, \zeta_{2}, \ldots, \zeta_{n}\right)^{T} \in E$ and a (possibly unknown) $\delta_{0}>0$ such that $f$ is a homeomorphism ${ }^{7}$ on the set $E_{\zeta, \delta_{0}} \triangleq$ $\prod_{i=1}^{n}\left[-\delta_{0}+\zeta_{i}, \delta_{0}+\zeta_{i}\right] \subset E$.

Remark 2. Assumption 2 holds if the function $f$ is continuously differentiable on $E_{\zeta, \delta_{0}}$ and its Jacobian matrix at $\zeta$ is invertible.

Remark 3. $\zeta$ and $\delta_{0}$ may not be unique.

We consider the two following systems in which $u$ is the input signal that is chosen by the designer to fit the identification goals, $y$ is the output that is accessible to measurements, $w$ is an internal signal not necessarily accessible to measurements, and $\nu$ is a noise not necessarily accessible to measurements. Wiener systems are characterized by the relations $\vartheta=u, \theta=w, y_{1}=f(w), y=y_{1}+\nu$; we denote by $\mathcal{H}_{w}$ the operator that maps $\left(u, x_{0}\right)$ to $y_{1}$ (see Figure 1 ). Hammerstein systems are characterized by the relations $w=f(u), \vartheta=w$ and $\theta=y_{1}, y=y_{1}+\nu$; we denote by $\mathcal{H}_{h}$ the operator that maps $\left(u, x_{0}\right)$ to $y_{1}$ (see Figure 2 ).

Our objective is to determine the function $f$ and the matrices $A, B, C, D$ from input/output data. Note that the determination of the unknowns can be done only up to an invertible matrix. That is, from input/output data it is only possible to determine $K f$ and $A, B K^{-1}, C, D K^{-1}$ where $K \in \mathbb{R}^{n \times n}$ is an arbitrary invertible matrix, for Hammerstein systems; and $f K$ and $A, B, K^{-1} C, K^{-1} D$ for Wiener systems. Without loss of generality, we take $K=G$. Thus, for Hammerstein systems, our objective will be the determination of the function $G f$ and matrices $A, B G^{-1}, C, D G^{-1}$ while for Wiener systems, our objective will be the determination of the function $f G$

\footnotetext{
7 A homeomorphism is a bijective continuous function whose inverse function is continuous. A homeomorphism is an open mapping.
} 
and matrices $A, B, G^{-1} C, G^{-1} D$. Note that the linear systems $\left(A, B G^{-1}, C, D G^{-1}\right)$ and $\left(A, B, G^{-1} C, G^{-1} D\right)$ have a static gain equal to the identity matrix $\mathcal{I}_{n}$ of $\mathbb{R}^{n \times n}$. Thus, without loss of generality, the identification problem can be transformed into the following equivalent one:

Problem statement: Determine the function $f$ and the matrices $A, B, C, D$ where Assumption 1 is replaced by:

Assumption 3. $G=\mathcal{I}_{n}$.

\section{IDENTIFICATION IN THE ABSENCE OF NOISE}

In this section, we assume that $\nu(t)=0, \forall t \geq 0$.

\subsection{First stage: Identification of the function $f$}

Let $\sigma \in E$ be given, and take as input signal $u(t)=$ $\sigma, \forall t \geq 0$. Consider first the case of the operator $\mathcal{H}_{w}$. Due to Assumption 3, it follows that $\lim _{t \rightarrow \infty} w(t)=$ $\sigma$. On the other hand, $y(t)=f(w(t)), \forall t \geq 0$, thus, using the continuity of $f$ we get that $\lim _{t \rightarrow \infty} y(t)=$ $\lim _{t \rightarrow \infty} f(w(t))=f(\sigma)$. Now, consider the operator $\mathcal{H}_{h}$, we have $w(t)=f(\sigma), \forall t \geq 0$ so that by Assumption 3 we have $\lim _{t \rightarrow \infty} y(t)=f(\sigma)$. Thus, the value $f(\sigma)$ is given in both cases by the relation

$$
f(\sigma)=\lim _{t \rightarrow \infty} y(t)
$$

Since the output $y$ is accessible to measurements, the value $f(\sigma)$ is determined. This process is repeated for a finite number of values of $\sigma$ which gives the unknown parameters $\rho_{i}, i=1, \ldots, N$.

Remark 4. It is usual in identification techniques to obtain an unknown element $\rho$ as $\lim _{t \rightarrow \infty} \hat{\rho}(t)$ where $\hat{\rho}$ is an estimate of $\rho$ at instant $t$. This means that we can obtain the exact value of $\rho$ only after an infinite time. However, in practice, the values $\hat{\rho}(t), t \geq T$ and $\rho$ can be set as close as desired by choosing $T$ large enough. This means that in the relation $\rho=\lim _{t \rightarrow \infty} \hat{\rho}(t)$ we need not to wait an infinite time to obtain the exact value of $\rho$ but we can get an approximation of $\rho$ with arbitrary precision by waiting a long enough finite time $T$. So, relation (5) implies that, in practice, after some finite time $T$, the value $f(\sigma)$ is obtained with a precision that depends on $T$. Since this experiment takes a finite time, it can be repeated for a finite number of values of $\sigma$.

\subsection{Second stage: Identification of the matrices $A, B, C$,} D

Let us consider the system described by Equations (1)(3) in which the input $\vartheta$ and output $\theta$ are accessible to measurements, and where the unknowns are the matrices $A, B, C, D$ to be determined from input-output data. This is a linear identification problem that has been studied extensively in the literature, see for example $[22,14,20,21,2]$ and references therein. Let $\mathcal{M}$ be some identification algorithm that allows the determination of the unknown matrices of the linear system, using some appropriate identification input $\vartheta$. We choose $\mathcal{M}$ with the additional property that the convergence properties of $\mathcal{M}$ do not change if the identification input $\vartheta$ is multiplied by a nonzero constant (this is the case for example for least-squares algorithms). Our objective is to show that the identification of Wiener or Hammerstein system can be reduced to that of identifying a linear system, which can be solved using the algorithm $\mathcal{M}$.

The function $f$ has been identified in Stage 1 so that we can obtain a known $\zeta \in E$, and a known $\delta_{0}>0$ such that, by Assumption 2, $f$ is invertible in $\prod_{i=1}^{n}\left[-\delta_{0}+\zeta_{i}, \delta_{0}+\zeta_{i}\right]$. Choose $0<\delta_{1}<\delta_{0}$ and define the following known compact set $\mathcal{K}_{1}=f\left(\prod_{i=1}^{n}\left[-\delta_{1}+\zeta_{i}, \delta_{1}+\zeta_{i}\right]\right)$. We proceed as follows.

Step 1: Preliminary experiment. Take $u(t)=\zeta, \forall t \geq$ 0 . Due to Assumption 3 and the continuity of $f$ we get that $\lim _{t \rightarrow \infty} w(t)=\zeta$ and $\lim _{t \rightarrow \infty} y(t)=f(\zeta)$ for the operator $\mathcal{H}_{w}$; and $w(t)=f(\zeta), \forall t \geq 0$, and $\lim _{t \rightarrow \infty} y(t)=f(\zeta)$ for the operator $\mathcal{H}_{h}$.

Step 2: Estimation of the linear subsystem parameters. Let $\vartheta$ be the identification input of the algorithm $\mathcal{M}$ for the determination of the unknown matrices $A, B$, $C, D$ of the linear system (1)-(3).

If we knew that the process is Wiener, we would use the following procedure $P_{w}$. Choose a series $\left(\varepsilon_{k} \in(0, \infty)\right)_{k \in \mathbb{N}}$ such that $\varepsilon_{k}>\varepsilon_{k+1}$ and $\lim _{k \rightarrow \infty} \varepsilon_{k}=0$.

(1) Take $k=1$

(2) Take $u(t)=\zeta+\varepsilon_{k} \vartheta(t), \forall t \geq 0$. If there exists some $t \geq 0$ for which $y(t) \notin \mathcal{K}_{1}$, then $k \leftarrow k+1$.

This algorithm has a finite number of loops as there exists some $\alpha$ that depends on $A, B, C, D$ such that $\|w-\zeta\|_{\infty} \leq \alpha\|u-\zeta\|_{\infty}$. That is, for some value of $k=k^{\star}$ we will have $\forall t \geq 0, y(t) \in \mathcal{K}_{1}$ which shows that $\forall t \geq 0, w(t) \in \prod_{i=1}^{n}\left[-\delta_{1}+\zeta_{i}, \delta_{1}+\zeta_{i}\right]$. Thus $w(t)$ can be computed from $y(t)$ using the relation

$$
w(t)=f^{-1}(y(t))
$$

Due to the linearity of the system (1)-(3), the identification algorithm $\mathcal{M}$ can now be applied to the input $\varepsilon_{k^{\star}} \vartheta=u-\zeta$ and its corresponding output $w-\zeta$ that is accessible to measurements due to Equation (6). As the convergence properties of $\mathcal{M}$ do not change if $\vartheta$ is multiplied by a nonzero constant (which is in this case $\varepsilon_{k^{\star}}$ ), it follows that we have determined an estimate $\left(A_{w}, B_{w}, C_{w}, D_{w}\right)$ of the system $(A, B, C, D)$.

If we knew that the process is Hammerstein, we would use the following procedure $P_{h}$. We have by Assumption 2 that the mapping $\mathrm{f}$ is open on $E_{\zeta, \delta_{0}}$. Thus, the set $\mathcal{K}_{1}$ contains an open ball $\mathcal{B}\left(f(\zeta), \delta_{3}\right)$ centered on $f(\zeta)$ with radius $\delta_{3}>$ 0 . Choose $u(t)=f^{-1}\left(f(\zeta)+\frac{\delta_{3}}{2\|\vartheta\|_{\infty}} \vartheta(t)\right), \forall t \geq 0$; this choice is possible as $f(\zeta)+\frac{\delta_{3}}{2\|\vartheta\|_{\infty}} \vartheta(t) \in \mathcal{B}\left(f(\zeta), \delta_{3}\right) \subset \mathcal{K}_{1}$ and $f$ is invertible by Assumption 2. Due to the linearity of the system (1)-(3), the identification algorithm $\mathcal{M}$ can now be applied to the input $\frac{\delta_{3}}{2\|\vartheta\|_{\infty}} \vartheta=f(u)-f(\zeta)$ and 
its corresponding output $y-f(\zeta)$. As the convergence properties of $\mathcal{M}$ do not change if $\vartheta$ is multiplied by a nonzero constant (which is in this case $\frac{\delta_{3}}{2\|\vartheta\|_{\infty}}$ ), it follows that we have determined an estimate $\left(A_{h}, B_{h}, C_{h}, D_{h}\right)$ of the system $(A, B, C, D)$.

However, we do not know whether the process is Wiener or Hammerstein. Thus we will apply both procedures $P_{w}$ and $P_{h}$ to the process. If the process is Wiener, and $P_{h}$ is applied, we may have the following scenarios:

(1) When applying the algorithm $\mathcal{M}$ to the input $f(u)-$ $f(\zeta)$ and the output $y-f(\zeta)$, the estimated parameters do not converge. If this happens we can be sure that the process is not Hammerstein.

(2) When applying the algorithm $\mathcal{M}$ to the input $f(u)-$ $f(\zeta)$ and the output $y-f(\zeta)$, the estimated parameters converge. This would happen, in particular, if the function $f$ is linear in the region $\mathcal{B}\left(f(\zeta), \frac{\delta_{3}}{2}\right)$.

In the second case, we obtain two models using the procedures $P_{w}$ and $P_{h}$. In this case, the identification method has a third step which is model validation. The same would happen is the process is Hammerstein and we apply $P_{w}$, that is we may be certain that the process is not Wiener, or get two models.

Step 3: Model validation. At this point two hypothetical models are available. One is of Wiener type, denoted $M_{w}$, and the other of Hammerstein type, denoted $M_{h}$ The two models have a common nonlinearity $f(\cdot)$, determined in Stage 1 of the identification procedure. They only differ by the linear subsystem model which is defined by $\left(A_{w}, B_{w}, C_{w}, D_{w}\right)$ for the first and $\left(A_{h}, B_{h}, C_{h}, D_{h}\right)$ for the second, along with the position of the linear system with respect to the nonlinearity. To determine the nature (Hammerstein or Wiener) of the true system, a prediction criteria based validation test is used. One applies a quite exciting input signal (e.g. a PRBS) to the system and collects the resulting output $y(t)$ over a sufficiently large interval, say $[0, T]$. Then, one applies the same input signal to both hypothetical models and collects the resulting outputs, respectively denoted $y_{w}(t)$ and $y_{h}(t)$. Compute the two mean square prediction errors:

In continuous-time: $J_{w}=\frac{1}{T} \int_{0}^{T}\left(y(t)-y_{w}(t)\right)^{2} \mathrm{~d} t$,

$$
J_{h}=\frac{1}{T} \int_{0}^{T}\left(y(t)-y_{w}(t)\right)^{2} \mathrm{~d} t
$$

In discrete-time: $\quad J_{w}=\frac{1}{T} \sum_{t=0}^{T}\left(y(t)-y_{w}(t)\right)^{2}$,

$$
J_{h}=\frac{1}{T} \sum_{t=0}^{T}\left(y(t)-y_{w}(t)\right)^{2} .
$$

If $J_{w}<J_{h}$ (resp. $J_{w}>J_{h}$ ), then the nature of the true nonlinear system under study is a Wiener (resp. Hammerstein) type, and the Wiener model $\left(A_{w}, B_{w}, C_{w}, D_{w}\right)$ (resp. Hammerstein model $\left.\left(A_{h}, B_{h}, C_{h}, D_{h}\right)\right)$ must be retained for the linear subsystem.

Note that in case the amplitude of the input signal is not large enough, the resulting output signal may not be very sensitive to the nonlinear effect of the system nonlinearity $f(\cdot)$. Then, the criteria $J_{w}$ and $J_{h}$ will be comparable making difficult the detection of the system nature. In this case, one must increase the amplitude of the input signal.

\section{IDENTIFICATION IN THE PRESENCE OF NOISE}

In this section the noise $\nu$ may not be identically zero.

Assumption 4. The stochastic process $\nu$ is zero mean and mean ergodic.

\subsection{Identification of the function $f$}

The identification methodology of Section 3.1 is modified as follows. Let $\sigma \in E$ be given, and take as input signal $u(t)=\sigma, \forall t \geq 0$. Consider first the case of the operator $\mathcal{H}_{w}$. Due to Assumption 3, it follows that $\lim _{t \rightarrow \infty} w(t)=$ $\sigma$. On the other hand, $y_{1}(t)=f(w(t)), \forall t \geq 0$, thus, using the continuity of $f$ we get that $\lim _{t \rightarrow \infty} y_{1}(t)=$ $\lim _{t \rightarrow \infty} f(w(t))=f(\sigma)$. Now, consider the operator $\mathcal{H}_{h}$, we have $w(t)=f(\sigma), \forall t \geq 0$ so that by Assumption 3 we have $\lim _{t \rightarrow \infty} y_{1}(t)=f(\sigma)$. We have $y(t)=y_{1}(t)+\nu(t)$ so that in steady-state we obtain $y(t)=f(\sigma)+\nu(t), \forall t \geq 0$. By Assumption 4 it follows that $\lim _{T \rightarrow \infty} \frac{1}{T} \int_{0}^{T} \nu(t) \mathrm{d} t=0$. Since the output $y$ is accessible to measurements, the value $f(\sigma)$ is determined by the relation ${ }^{8}$

$$
f(\sigma)=\lim _{T \rightarrow \infty} \frac{1}{T} \int_{0}^{T} y(t) \mathrm{d} t
$$

This process is repeated for a finite number of values of $\sigma$ which gives the unknown parameters $\rho_{i}, i=1, \ldots, N$.

\subsection{Identification of the matrices $A, B, C, D$}

As the function $f$ has been identified on $E$, the identification methodology follows as in Section 3.2 and consists in transforming the identification problem for Hammerstein or Wiener systems into that of identification of a linear system in the presence of noise. The latter problem has been studied extensively in the literature and numerous algorithms exist to solve it $[22,14,20,21,2]$. This means that using any of these algorithms ${ }^{9}$ solves the problem of determining the matrices $A, B, C, D$.

\section{CONCLUSION}

This paper has presented a simple algorithm for identifying a wide class of Hammerstein and Wiener systems. The framework is valid for SISO and MIMO systems, and also for discrete-time and continuous-time systems. The algorithm has three main steps. The first step consists in

8 If time is discrete, the symbol $\int$ is replaced by $\sum$

9 under the condition that the convergence properties of the algorithm do not change if the identification input is multiplied by a nonzero constant. 
identifying the static nonlinearity. The second step consists in transforming the Hammerstein or Wiener identification problem into a linear identification problem for which standard methods exist. The second step may lead to two models, in which case a third step of model validation is applied. The main contributions of the paper is that we only need to know that the system belongs to the set $\{$ Wiener, Hammerstein $\}$; and that SISO and MIMO systems, along with continuous and discrete-time settings are dealt within the same framework.

\section{REFERENCES}

[1] R. A. Adams, J. J. F. Fournier. Sobolev Spaces. Elsevier, 2003.

[2] D. Bauer. "Asymptotic properties of subspace estimators," Automatica, vol. 41, pp. 359-376, 2005.

[3] E. W. Bai. "Frequency domain identification of Wiener models," Automatica, vol. 39, pp. 1521-1530, 2003.

[4] S.I. Biagiola, J.L. Figueroa. "Wiener and Hammerstein uncertain models identification," Math. Comp. Simul., vol. 79, pp. 3296-3313, 2009.

[5] K. H. Chan, J. Bao, W. J. Whiten. "Identification of MIMO Hammerstein systems using cardinal spline functions," J. Process Cont., vol. 16, pp. 659-670, 2006.

[6] H. F. Chen. "Recursive identification for Wiener model with discontinuous piecewise linear function," IEEE Trans. Aut. Cont., vol. 51, no. 3, pp. 390-400, 2006.

[7] V. Cerone, D. Regruto. "Parameter bounds for discrete-time Hammerstein models With bounded output errors," IEEE Transactions on Automatic Control, vol. 48(10), pp. 1855-1860, 2003.

[8] E. J. Dempsey, D. T. Westwick. "Identification of Hammerstein models with cubic spline nonlinearities," IEEE Trans. Biomed. Eng., vol. 51, no. 2, pp. 237-245, 2004.

[9] G. Dolanc, S. Strmčnik. "Identification of nonlinear systems using a piecewise -linear Hammerstein model," Syst. Cont. Letters., vol. 54, no. 2, pp. 145$158,2005$.

[10] W. Greblicki. "Nonlinearity recovering in Wiener system driven with correlated signal," IEEE Trans. Aut. Cont., vol. 49, no. 10, pp. 1805-1810, 2004.

[11] F. Giri, E-R Bai. "Block-Oriented Nonlinear System Identification," Lecture Notes in Control and Information Sciences, Springer-Verlag Berlin, 2010.

[12] F. Giri, Y. Rochdi, A. Brouri, F.Z. Chaoui. "Parameter identification of Hammerstein systems containing backlash operators with arbitrary-shape parametric borders," Automatica, vol. 47, pp. 1827-1833, 2011.

[13] F. Giri, Y. Rochdi, F.Z. Chaoui. "An analytic geometry approach to Wiener system frequency identification," Trans. Aut. Cont., vol. 54, no. 4, pp. 683-696, 2009.

[14] L. Ljung. System Identification. Theory for the User. Second Edition, Prentice Hall, Upper Saddle River, NJ, 1999.

[15] S. Lakshminarayanan, S.L. Shah, K. Nandakumar. "Identification of Hammerstein models using multivariate statistical tools," Chem. Eng. Science. vol. 50, no. 22, pp. 3599-3613, 1995.
[16] K. S. Narendra, P. G. Gallman. "An iterative method for the identification of nonlinear systems using a Hammerstein model," IEEE-Transactions on Automatic Control, pp. 546-550, July 1966.

[17] A. E. Nordsjö, L. H. Zetterberg. "Identification of certain time-varying nonlinear Wiener and Hammerstein systems," IEEE Trans. Signal Proc., vol. 49, no. 3, pp. 577-592, 2001.

[18] W. Rudin. Real and Complex Analysis. McGraw-Hill Series in Higher Mathematics, Third Edition, USA, 1987.

[19] S. W. Sung. "System identification method for Hammerstein processes," Indust. Eng. Chemist. Research., vol. 41, no. 17, pp. 4295-4302, 2002.

[20] H. Unbehauen, G.P. Rao. "Continuous-time approaches to system identification- a survey" Automatica, vol. 26, no. 1, pp. 23-35, 1990.

[21] M. Verhaegen. "Identification of the deterministic part of MIMO state space models given in innovations form from input-output data" Automatica, vol. 30, no. 1, pp. 61-74, 1994.

[22] Y. Zhu. Multivariable System Identification for Process Control. Pergamon-Elsevier, Oxford, UK, 2001. 